TRABAJO ORIGINAL

\title{
IMPACTO DE LA CIRUGÍA BARIÁTRICA EN PACIENTES CON OBESIDAD Y DIABETES MELLITUS TIPO 1
}

\author{
IMPACT OF BARIATRIC SURGERY IN PATIENTS \\ WITH OBESITY AND TYPE 1 DIABETES MELLITUS
}

Paola Poloํ, María Graciela Álvarez², Eduardo Babor ${ }^{3}$, Maia Herrero $^{4}$, Sandra González ${ }^{5}$, María Yuma ${ }^{6}$, Isaac Sinay ${ }^{7}$, León Litwak ${ }^{8}$, Susana Gutt ${ }^{9}$, Susana Fuentes ${ }^{10}$, Carla Musso ${ }^{11}$, Marianela Aguirre Ackermann ${ }^{12}$

\section{RESUMEN}

Introducción: la obesidad es un problema médico serio y en crecimiento en los pacientes con diabetes mellitus tipo 1 (DM1). El tratamiento de reemplazo con insulina es la única terapia disponible y los principales efectos adversos asociados son la hipoglucemia y la ganancia de peso. La cirugía bariátrica (CB) mostró mejoría en el control glucémico en los pacientes con DM2 pero se han reportados pocos casos en DM1.

Objetivos: evaluar los cambios en los parámetros metabólicos en pacientes con obesidad y DM1 a quienes se les realizó una CB.

Materiales y métodos: en forma retrospectiva se evaluaron las historias clínicas de siete pacientes con DM1 y obesidad que fueron tratados con CB. Se utilizó el test de Wilcoxon para muestras apareadas a fin de evaluar la diferencia entre los datos pre cirugía y al año de la misma.

Resultados: se evaluaron siete pacientes con DM1. La media de edad fue de 48 años (IQR 45 a 49). La media de índice de masa corporal basal y al año fue $39,6 \mathrm{Kg} / \mathrm{m}^{2}$ (IQR 35 a 42) y $24,9 \mathrm{Kg} / \mathrm{m}^{2}$ (IQR 24,7 a 29,5 ) respectivamente. La media de hemoglobina glicosilada basal y al año de seguimiento fue de $9,3 \%$ (IQR 8,3 a 10,5 ) y 7,2\% (IQR 6,7 a 8,8). La media del requerimiento de insulina antes y después de la CB fue de $110 \mathrm{UI}$ (IQR 70 a 120) y 24 UI (IQR 16 a 30). Todas las diferencias fueron estadísticamente significativas $(p<0,05)$.

Conclusiones: los hallazgos de este estudio alientan a considerar a la CB como una herramienta en los pacientes con DM1 y obesidad con el objetivo de mejorar el control glucémico y el peso corporal.

Palabras clave: diabetes mellitus tipo 1; obesidad, cirugía bariátrica.

Revista de la Sociedad Argentina de Diabetes 2019; Vol. 53 (139-142)

\section{ABSTRACT}

Introduction: obesity is a serious growing medical problem in type 1 diabetes mellitus (DM1). Insulin replacement is the only available therapy for these patients with DM1 and the main problems associated with it are hypoglycemia and weight gain. Bariatric surgery (BS) showed improvement in glycemic control in type 2 but only few cases of DM1 have been reported.

Objectives: to evaluate changes in metabolic parameters in obese DM1 patients who underwent BS.

Materials and methods: retrospectively, the clinical histories of seven patients with DM1 and obesity who were treated with $C B$ were evaluated. The Wilcoxon test was used for paired samples in order to assess the difference between the pre-surgery data and one year after it.

Results: seven patients DM1 were evaluated. The median age was 48 years (IQR 45 to 49). The median body mass index at baseline and at follow-up were $39.6 \mathrm{Kg} / \mathrm{m}$ (IQR 35 to 42) and $24.9 \mathrm{Kg} / \mathrm{m}^{2}$ (IQR 24.7 to 29.5) respectively. The median glycated hemoglobin at baseline and at follow-up were 9.3\% (IQR 8.3 to 10.5) and $7.2 \%$ (IQR 6.7 to 8.8) respectively. The median insulin requirements before and after $B S$ were 110 UI (IOR 70 a 120) and 24 UI (IQR 16 a 30). All differences were statistically significant ( $p$ value $<0.05$ ).

Conclusions: our findings encourage considering BS as a tool in type 1 obese diabetic patients in terms to improve management of glycemic control and body weight.

Key words: diabetes mellitus type 1; obesity; bariatric surgery.

Revista de la Sociedad Argentina de Diabetes 2019; Vol. 53 (139-142) 
1 Médica Endocrinóloga, Servicio de Endocrinología, Sanatorio Las Lomas, Provincia de Buenos Aires, Argentina

2 Médica especialista en Medicina Interna y Nutrición, Coordinadora del Equipo del Centro de Estudio y Tratamiento de Obesidad Severa (CETOS), Ciudad Autónoma de Buenos Aires, Argentina

3 Médico Cirujano especialista en Cirugía Laparoscópica y Bariátrica, Centro de Estudio y Tratamiento de Obesidad Severa (CETOS), Ciudad Autónoma de Buenos Aires, Argentina

4 Médica Endocrinóloga, Sanatorio del Salvador, Centro Médico Marcheggiani y Hospital Dr. Ángel Ferreyra, Córdoba, Argentina

5 Médica Clínica especializada en Diabetes, Clínica IMA, Provincia de Buenos Aires, Argentina

6 Médica especialista en Nutrición, Hospital Italiano de Buenos Aires, Ciudad Autónoma de Buenos Aires, Argentina

7 Médico Endocrinólogo, consultor del Insitituto Cardiovascular de Buenos Aires, Ciudad Autónoma de Buenos Aires, Argentina

8 Médico especialista en Endocrinología, consultor del Servicio de Endocrinología del Hospital Italiano de Buenos Aires, Ciudad Autónoma de Buenos Aires, Argentina
9 Médica especialista en Nutrición, Jefa de Nutrición, Hospital Italiano de Buenos Aires, Ciudad Autónoma de Buenos Aires, Argentina

10 Médica especialista en Medicina Interna y Diabetes, Coordinadora del Comité de Obesidad de la Sociedad Argentina de Diabetes, Ciudad Autónoma de Buenos Aires, Argentina

11 Médica Endocrinóloga, Coordinadora de Diabetes, Fundación Favaloro, Ciudad Autónoma de Buenos Aires, Argentina

12 Médica especialista en Medicina Interna y Nutrición, Coordinadora de Nutrición y Diabetes del Centro Integral de Endocrinología y Nutrición (CIEN), Ciudad Autónoma de Buenos Aires, Argentina

\section{Contacto de la autora: Paola Polo}

E-mail: polopaolag@gmail.com

Correspondencia: Pisco $1223^{\circ}$ UF 307, Pilar, Provincia de

Buenos Aires, Argentina

Fecha de trabajo recibido: 24/06/19

Fecha de trabajo aceptado: 27/11/19

Conflictos de interés: los autores declaran que no existe conflicto de interés

\section{INTRODUCCIÓN}

Actualmente el $50 \%$ de los pacientes con diabetes mellitus tipo 1 (DM1) tiene obesidad o sobrepeso, y entre el 20 y el $40 \%$ cumplen los criterios de síndrome metabólico, según población y criterios diagnósticos utilizados.

El tratamiento intensificado con insulina asociado al conteo de hidratos de carbono y la ausencia de educación diabetológica adecuada incrementan la prevalencia de obesidad en esta población'. El Diabetes Control and Complications Trial (DCCT) mostró una excesiva ganancia de peso en el subgrupo de pacientes con tratamiento intensificado, con un aumento del índice cintura/cadera y de los niveles de triglicéridos, colesterol LDL y apolipoproteina $\mathrm{B}$, y disminución del colesterol $\mathrm{HDL}$, sugiriendo un aumento de la grasa intrabdominal ${ }^{2,3}$. Estudios posteriores confirmaron que la ganancia de peso en estos pacientes resultó en una dislipemia similar a la del síndrome metabólico, con su consecuente aumento en el riesgo cardiovascular ${ }^{4}$.

Por otro lado, el tratamiento de la DM1 en individuos con obesidad severa puede ser un desafío, con posibles efectos anabólicos de la terapia intensiva con insulina sobre el peso corporal, que exacerba aún más la resistencia a la insulina $a^{5,6}$.

Algunos estudios demostraron que la obesidad y el síndrome metabólico en la DM1 se asocian con el desarrollo y la progresión de las complicaciones micro y macrovasculares 7,8 .

La cirugía bariátrica (CB) mostró en múltiples trabajos randomizados y controlados mejoría en la glucemia y en el control de peso en pacientes con
DM2, sin embargo se publicaron pocos artículos que analizan el efecto de la misma en la DM1 y sus resultados no han sido uniformes ${ }^{9,10,11}$. Los diseños retrospectivos, los volúmenes de muestra pequeños y los cortos tiempos de seguimiento en la mayoría son limitaciones para los estudios de casos observacionales publicados.

El objetivo de nuestro estudio fue evaluar el impacto de la CB sobre el peso y los parámetros metabólicos en pacientes con DM1.

\section{MATERIALES Y MÉTODOS}

Realizamos una revisión retrospectiva de las historias clínicas de siete pacientes con DM1 y obesidad a quienes se les efectuó una cirugía bariátrica. Los pacientes tenían diagnóstico de obesidad grado II o III previo a la intervención. El diagnóstico de DM1 se confirmó en todos los pacientes por la presencia de pancreatitis autonimune, ausencia de péptido- $\mathrm{C}$ o historia de cetoacidosis diabética. Se evaluó a los pacientes basal y al año de realizada la cirugía.

En tres pacientes se realizó bypass gástrico en $Y$ de Roux y gastrectomía en manga en cuatro. Uno de ellos tenía, además, diagnóstico de enfermedad celíaca.

Se empleó el test de Wilcoxon para muestras apareadas a fin de evaluar la diferencia entre los datos pre cirugía y al año de la misma.

\section{RESULTADOS}

Se evaluaron siete pacientes con DM1. La media de edad fue de 48 años (IQR 45 a 49); todos 
fueron mujeres. La media de índice de masa corporal basal y al año fue $39,6 \mathrm{Kg} / \mathrm{m}^{2}$ (IQR 35 a 42) y $24,9 \mathrm{Kg} / \mathrm{m}^{2}$ (IOR 24,7 a 29,5 ) respectivamente.

La media de hemoglobina glicosilada basal y al seguimiento fue de $9,3 \%$ (IOR 8,3 a 10,5 ) y $7,2 \%$ (IOR 6,7 a 8,8) respectivamente. La media del requerimiento de insulina antes y después de la $C B$ fue $110 \mathrm{UI}$ (IQR 70 a 120) y 24 UI (IQR 16 a 30). Todas las diferencias fueron estadísticamente significativas $(p<0,05)$.

Con respecto a las complicaciones postquirúrgicas, una paciente presentó estenosis de la anastomosis al mes de la cirugía, que se resolvió con dilatación endoscópica, mientras que dos pacientes padecieron hipoglucemias moderadas en los primeros sesenta días que se corrigieron al bajar las dosis de insulina.

\begin{tabular}{|l|l|l|c|}
\hline & \multicolumn{1}{|c|}{ Pre cirugía } & \multicolumn{1}{c|}{ Post cirugía } & \multicolumn{1}{c|}{ P } \\
\hline $\mathrm{IMC}\left(\mathrm{kg} / \mathrm{m}^{2}\right)$ & 39,6 IQR 35 a 42 & 24,9 IQR 24,7 a 29,5 & $<0,05$ \\
\hline $\mathrm{HbA1c} \%$ & $9,3 \%$ IQR 8,3-10,5 & $7,2 \%$ IQR 6,7 a 8,8 & $<0,05$ \\
\hline $\begin{array}{l}\text { Requerimientos } \\
\text { de insulina }\end{array}$ & $110 \mathrm{UI}$ IQR 70-120 & 24UI IQR 16-30 & $<0,05$ \\
\hline $\mathrm{COL}$ & $208(105-282)$ & $165,5(114-200)$ & $<0,05$ \\
\hline $\mathrm{HDL}$ & $46(40-45)$ & $56,8(45-71)$ & $<0,05$ \\
\hline TA & $136,8(120-160)$ & $121,1(100-130)$ & $<0,05$ \\
\hline
\end{tabular}

Tabla 1: Características basales y postquirúrgicas $(\mathrm{N}=7)$.

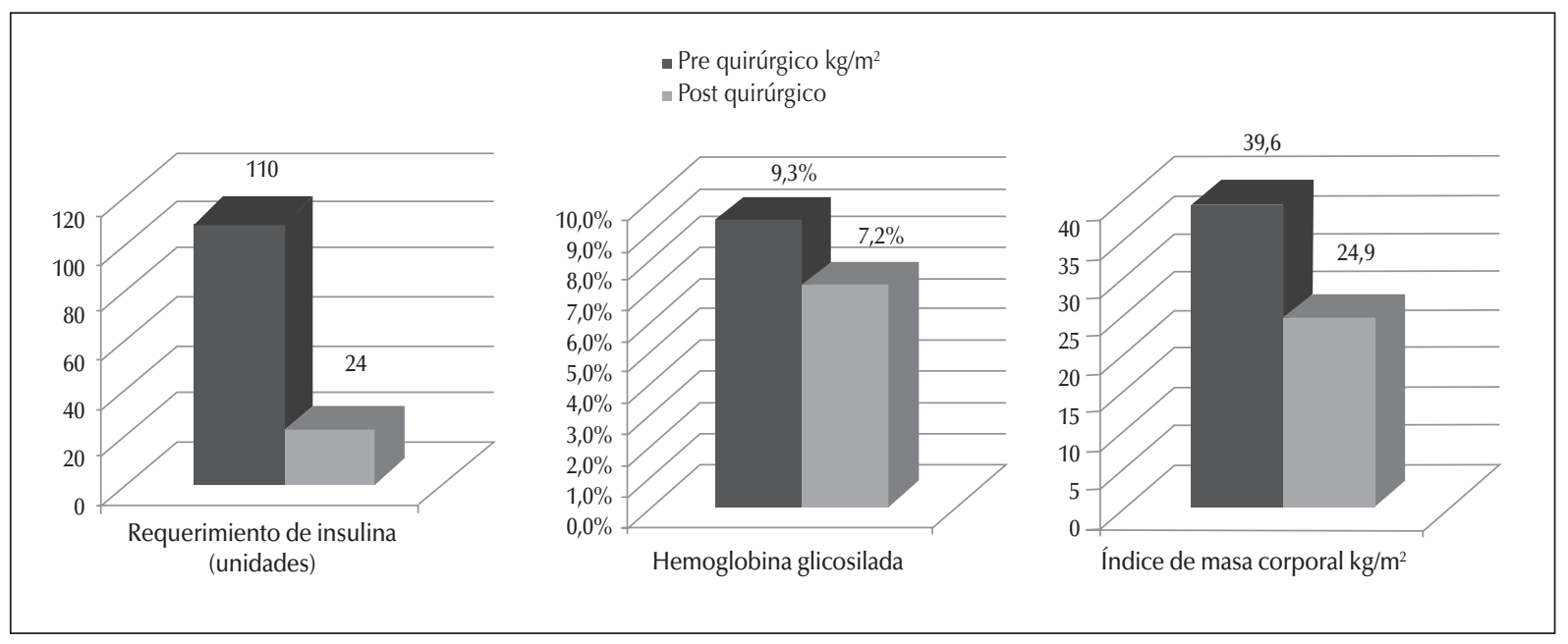

Figura 1: Requerimiento de insulina, $\mathrm{HbA1c}$ e IMC.

\section{DISCUSIÓN}

La creciente prevalencia de obesidad en el mundo también se extiende a la población de pacientes con DM1. Las indicaciones y beneficios de la CB en DM2 están bien establecidas, sin embargo no existe hasta el momento consenso en indicaciones y beneficios en pacientes con DM1.

El resultado del presente estudio muestra que los pacientes con obesidad y DM1 que se realizan CB -bypass gástrico o manga gástrica- presentan significativa mejoría tanto en IMC como en parámetros metabólicos (glucemia, hemoglobina glicosilada, colesterol total, colesterol LDL y HDL) y requerimientos de insulina, coincidente con lo previamente descripto por otros autores ${ }^{12}$. Específicamente con respecto al control glucémico, nuestro grupo de pacientes tuvo una mejoría en la glucemia mensurada por una disminución promedio de 2,1 puntos en la HbA1c.

La revisión de CB en pacientes con DM1, que incluyó mayor número de pacientes, la publicaron Kirwan et al. ${ }^{13}$. Allí se incluyeron 17 estudios con 107 individuos, con al menos un año de seguimiento postoperatorio. Todos los estudios describieron una reducción significativa en el peso corporal. Se evidenció un notable descenso en los requerimientos diarios de insulina ajustados por peso en todas las series de casos, excepto una. Sin embargo, los cambios de HbA1c no fueron consistentes lo que podría explicarse en parte porque los $\mathrm{N}$ pequeños no tenían el poder suficiente para mostrar cambios postoperatorios en la HbA1c. Además, las diferencias en el diseño de los estudios, las características de los pacientes y 
los procedimientos quirúrgicos entre los estudios podrían explicar las diferencias informadas en los cambios de $\mathrm{HbA} 1 \mathrm{c}$.

¿Qué ocurre en el largo plazo? Un trabajo que evaluó los resultados a corto y largo plazo de la CB en pacientes con obesidad y DM1, y los comparó con pacientes con obesidad y DM1 que no se realizaron $\mathrm{CB}^{14}$, encontró que si bien a corto plazo la CB fue exitosa en el control del peso y la glucosa, a largo plazo la CB fue exitosa sólo en el control de la obesidad, no así en el control de los parámetros glucémicos.

Otro estudio ${ }^{15}$-que evaluó a 32 pacientes con DM1 seguidos durante 4,6 años, con seguimiento anual de IMC, HbA1c, requerimientos de insulina, evolución de las comorbilidades y complicaciones microvasculares- halló que la CB en pacientes con DM1 proporcionó principalmente beneficios en la reducción del peso, requerimientos de insulina, comorbilidades de la obesidad y algunos beneficios en las complicaciones de la DM, pero el efecto en el control glucémico a largo plazo fue mínimo.

En resumen, los estudios a largo plazo demuestran que se mantienen los efectos de la CB sobre el peso y las comorbilidades de la obesidad y los requerimientos de insulina, pero no se mantienen los beneficios del control glucémico observados en el corto plazo de la CB.

\section{CONCLUSIONES}

Dado el aumento en la incidencia de obesidad severa en los pacientes con DM1, se espera que la utilización de CB en pacientes con obesidad y DM1 sea cada vez mayor.

Los hallazgos de nuestra serie de casos alientan a considerar a la CB como una herramienta terapéutica efectiva en pacientes con DM1 y obesidad, con el objetivo de mejorar el control glucémico y lograr el descenso del peso corporal. Dado que el seguimiento evaluado en estos pacientes fue a un año, se necesitaría un mayor seguimiento para analizar si la mejoría evidenciada en el control metabólico se sostiene a largo plazo.

A la luz de los resultados, sería apropiado considerar a la CB como una opción terapéutica eficaz en pacientes con obesidad y DM1, siendo el principal beneficio el manejo del peso corporal y la consecuencia de la mejoría del peso corporal en los parámetros metabólicos que se derivan de ella. Los resultados a corto plazo de la CB en pacientes con DM1 son alentadores, pero se necesitan estudios con un número más grande de pacientes y mayor seguimiento.

\section{BIBLIOGRAFÍA}

1. Chillarón JJ, Flores Le-Roux JA, Benaiges D, Pedro-Botet J. Type 1 diabetes, metabolic syndrome and cardiovascular risk. Metabolism 2014; 63:181-187.

2. DCCT Reserch Group. The absence of a glycemic threshold for the development of long-term complications: the perspective of the Diabetes Control and Complications Trial. Diabetes 1996; 45:1289-1298.

3. Buchwald H, Estok R, Fahrbach K, Banel D, Jensen MD, Pories WJ, Bantle JP, Sledge I. Weight and type 2 diabetes after bariatric surgery: systematic review and meta-analysis. Am J Med 2009; 122 (3): 248-56.

4. Sibley SD, Palmer JP, Hirsch IB, Brunzell. Visceral obesity, hepatic lipase activity and dyslipidemia in type 1 diabetes. J Clin Endocrinol Metab 2003 Jul; 88(7).

5. Franck M, Paquot N, Scheen AJ. Influence of body weight on the natural history of and the therapeutic approaches to type 1 diabetes. Rev Med Liege 2012; 67:461-467.

6. Cohen R. Comment on: should metabolic surgery be offered in morbidly obese patients with type I diabetes? Surg Obes Relat Dis 2015; $11: 805-807$

7. Price SA, Gorelik A, Fourlanos S, Colman PG, Wentworth JM. Obesity is associated with retinopathy and macrovascular disease in type 1 diabetes. Obes Res Clin Pract 2014; 8:e178-e182.

8. De Block CE, De Leeuw IH, Van Gaal LF. Impact of overweight on chronic microvascular complications in type 1 diabetic patients. Diabetes Care 2005; 28:1649-1655

9. Czupryniac L, Wiszniewski M, Szymansky D. Long term results of gastric bypass surgery in morbidly obese type 1 diabetes patients. Obes Surg 2010; 20:506-8.

10. Mendez CE, Tanenberg RJ, PoriesW. Outcomes of Roux enY gastric bypass surgery for severely obese patients with type 1 diabetes: a case series report. Diabetes Metab Syndr Obes 2010; 3:281-283.

11. Brethauer S, Aminian A, Rosental R. Bariatric surgery improves the metabolic profile of morbidly obese patients with type 1 diabetes. Diabetes Care 2014; 37: e51-e52.

12. Lannoo M, Dillemans B, Van Nieuwenhove $Y$. Bariatric surgery induces weight loss but does not improve glycemic control in patients with type 1 diabetes. Diabetes Care 2014; 37: e173-e174.

13. Kirwan JP, Aminian A, Kashyap S. Bariatric surgery in obese patients with type 1 diabetes. Diabetes Care 2016; 39:941-948.

14. Landau Z, Kowen-Sandbank G, Jakubowicz D. Bariatric surgery in patients with type 1 diabetes: special considerations are warranted. Ther Adv Endocrinol Metab 2019; Vol. 10: 1-8.

15. Vilarrasa N, Rubio MA, Miñambres I. Long-term outcomes in patients with morbid obesity and type 1 diabetes undergoing bariatric surgery. Obesity Surgery 2017 Apr; 27(4):856-863. 